\title{
Observability Analysis by Poincaré Normal Forms
}

\author{
L.Boutat-Baddas ${ }^{1}$, D. Boutat ${ }^{2}$ and J.P. Barbot ${ }^{3}$ \\ ${ }^{1}$ CRAN-CNRS (UMR 7039), Nancy University, IUT de Longwy, 186, rue de Lorraine, 54400 Cosnes-et-Romain. \\ latifa.boutat-baddas@iut-longwy.uhp-nancy.fr \\ ${ }^{2}$ ENSI-Bourges, Institut PRISME/ 10, Bd. Lahitolle, 18020 Bourges Cedex. \\ driss.boutat@ensi-bourges.fr \\ 3 Equipe Commande des Systèmes (ECS), ENSEA EPI-ALIEN INRIA , 6 Av. du Ponceau, 95014 Cergy Cedex. \\ barbot@ensea.fr
}

\begin{abstract}
This paper deals with quadratic equivalence, normal forms of observability, characteristic matrices and normal quadratic numbers for nonlinear Single-Input Single-Output (SISO) systems. We investigated both cases; nonlinear systems linearly observable and nonlinear systems with one linear unobservable mode. Particularly, the effect of the normal quadratic numbers on the observer design is pointed out. Finally, a faster observability analysis is proposed using characteristic matrices and normal quadratic numbers. Throughout the paper, academic examples as well as bio-reactor example highlight our purpose.
\end{abstract}

Key Words: Observability, Poincaré's normal form, observability singularity.

\section{Introduction}

The fact of being able to write down explicit solutions for linear differential equations allows a complete analysis of their behaviors. For this reason, the linearization problem of nonlinear systems, at least in the neighborhood of a singular point, is one approach for studying nonlinear systems. A rigorous mathematical technique which allows substantial progress in this approach is the normal form method. This method was first addressed by Poincaré (see [P]). Poincaré's theorem, which applies to analytic systems, shows that when certain non-resonance conditions are satisfied there is an analytic change of coordinates which transforms a nonlinear system into a linear one. For linearly controllable and uncontrollable systems, homogeneous approximation was first addressed by A. Krener in [K]. His idea is to apply Poincaré's technique by using not only a diffeomorphism but also a state feedback. Lately, linearization and Poincaré's method have been used for observability problems. More precisely, consider the following system:

$$
\left\{\begin{array}{l}
\dot{x}=f(x) \\
y=h(x)
\end{array}\right.
$$

where vector fields $f: \mathbb{R}^{n} \rightarrow \mathbb{R}^{n}$ and $h: \mathbb{R}^{n} \rightarrow$ $\mathbb{R}^{m}$ are assumed to be smooth where $f(0)=0$ and $h(0)=0$.

The observability problem is whether we can estimate the current state $x(t)$ from past observations $y(s), s \leq t$ without measuring all state variables. It is well-known that, by appropriate signal processing, we are often able to obtain good estimates of all state variables from measured outputs. The algorithm that performs this signal processing is called an observer. In the case of observable linear systems: $f(x)=A x$ and $h(x)=C x$ where $A \in \mathbb{R}^{n \times n}$ and $C \in \mathbb{R}^{m \times n}$ coupling the internal model and the innovation correction leads to the observer equations:

$$
\left\{\begin{array}{l}
\dot{\hat{x}}=A \widehat{x}+K(y-\widehat{y}) \\
\widehat{y}=C \widehat{x}
\end{array}\right.
$$

where $\widehat{x}$ is the estimate state and $\widehat{y}$ is the expected observation, $K$ is the observer gain matrix. The estimate error $e:=x-\widehat{x}$ is driven by the following equation:

$$
\dot{e}=(A-K C) e .
$$

If the pair $(A, C)$ is observable, then we can choose the observer poles of $A-K C$ such that $: e(t) \rightarrow 0$ as $t \rightarrow+\infty$.

Motivated by these considerations, the observer linearization problem was raised. Is it possible to find a state neighborhood $U$ of 0 in $\mathbb{R}^{n}$, and a change of state coordinates $z=\theta(x)$ such that dynamic (1) is linear driven by nonlinear output-injection:

$$
\dot{z}=A z-\beta(y) .
$$

where $\beta: \mathbb{R}^{m} \rightarrow \mathbb{R}^{n}$ is a smooth vector field. Note that the output-injection term is canceled in the observation error dynamic for system (2). 
The diffeomorphism $\theta$ must satisfy the following first-order partial differential equation:

$$
\frac{\partial \theta}{\partial x}(x) f(x)=A \theta(x)-\beta(h(x)) .
$$

Krener and Isidori in $[\mathrm{KI}]$ showed that equation (3) has a solution if and only if the following two conditions are satisfied:

i) the codistribution $\operatorname{span}\left\{d h, d L_{f} h, \ldots, d L_{f}^{n-1} h\right\}$ is of rank $n$ at 0 ,

ii) $\left[\tau, a d_{f}^{k} \tau\right]=0$ for all $k=1,3, \ldots, 2 n-1$ where $\tau$ is the vector field which fulfils the following equations:

$$
\begin{aligned}
d L_{f}^{k} h(\tau) & =0 \text { for } 0 \leq k \leq n-2 \\
d L_{f}^{n-1} h(\tau) & =1
\end{aligned}
$$

Moreover in [KR], Krener and Respondek relax the above conditions by yielding a diffeomorphism on the output. Precisely, if

Condition $1 i$ ) For $n$ even, it is necessary that for $k=1: n-1$

$$
\begin{aligned}
& {\left[\tau_{1}, \tau_{k}\right]=0 \text { for } k=1: n-1 \text { and }} \\
& {\left[\tau_{1}, \tau_{n}\right]=\alpha(y) \tau_{1}}
\end{aligned}
$$

ii) For $n$ odd, it is necessary that for $k=1: n-1$

$$
\begin{aligned}
& {\left[\tau_{1}, \tau_{n}\right]=\left[\tau_{1}, \tau_{k}\right]=\left[\tau_{2}, \tau_{k}\right]=0 \text { and }} \\
& {\left[\tau_{2}, \tau_{n}\right]-\alpha(y) \tau_{1} \in \operatorname{Span}\left(\tau_{1}\right)}
\end{aligned}
$$

these conditions enable us to find a local diffeomorphism on the output $\mathfrak{F}(y)$. Then, we consider the following dynamic system:

$$
\left\{\begin{array}{l}
\dot{x}=f(x) \\
\bar{y}=\mathfrak{F}(y)
\end{array}\right.
$$

where the dynamic is the same as in (1) and the output replaced by $\mathfrak{F}(y)$. Therefore, we say that system (1) is linearizable in the sense of Krener-Respondek if and only if system (4) is linearizable in the sense of Krener-Isidori.

Remark 2 This paper deals with system (1) when the Condition 1 is not verified and with system (4) when the Condition 1 is verified. Consequently, the proposed approach considers systems (1) or (4) which is not exactly linearizable.

An analytical approach to solve equation (3) is used by Kazantzis and Kravaris [KK2]. They considered the restricted form of this problem, where the outputinjection is linear, $\beta(y)=B y$. They showed, using a particular form of the Lyapunov Auxiliary Theorem [L] that (3) has a unique solution under certain assumptions. Recently, under very general conditions and for $\beta(y)$ unspecified, Krener and Xiao [KX] proved the existence and uniqueness of this solution. This allows us to design an observer for a larger class of nonlinear systems.

In this paper and in the preliminary version [BBBT1], by using quadratic transformations modulo input-output injection, we put linearly observable and linearly unobservable in one direction systems in particular normal forms. In particular, we are interested in the characterization of quadratic equivalence modulo input-output injection of systems for which we can design an observer. We show that every system lies in an equivalence class and is characterized by a list of relatively easy computable numbers. Rigorously speaking, our problem statement is as follows:

1) How can we characterize the fact that a system is quadratically linearizable modulo an input-output injection? If it is not the case, then what is its quadratic normal form?

The answer to both questions is given in Theorem 12 for nonlinear systems linearly observable and in Theorem 14 for nonlinear systems with one linear unobservable mode.

2) How can we compute normal quadratic numbers, i.e. quadratic coefficients which are not equal to zero in the quadratic normal form?

The answer to this question is given in Proposition 21 for nonlinear systems linearly observable and in Proposition 27 for nonlinear systems with one linear unobservable mode.

Our motivations to use this kind of observability normal forms are:

If the linear approximation is observable the proposed observability normal form highlights the structural obstruction to transform the system in well known injection form. Obviously the approximation is only valid locally, but thanks to this form, it is possible to analyse which type of obstructions we have. Nevertheless, the main interest of the proposed observability normal form is when the linear approximation is unobservable, in this case thanks to the normal form and more particularly due to the resonant terms it is possible to determine if or not the system is observable thanks to high order terms (see Remark 15). So from this analysis in the paper it is shown that it is possible to design an observer (obviously only locally valid). We think that such observability bifurcation (or more simply observability singularity) may be very well analysed by the observabilty normal form and this not only at the point $x=0$ and $u=0$ but around all observabilty singularity point thanks 
to a coordinate change and output set up. The study of such normal form around a manifold of observabilty singularity must be one of our future works. Nevertheless, the possibility to design an observer of the same dimension as that of the system, instead of searching for the information in the extra derivative (see the definition of weakly locally observability), is to keep the consistence with respect to a global observer design (we have just to switch off such observer close to the singularity, but without dimension problem). The normal form may be also used to analyse if the system stays or not in observability singularity domain.

The paper is organized as follows. Notations and the definition of the quadratic equivalence modulo an input-output injection are presented in section 2. In section 3 , we give homological equations and quadratic normal forms for nonlinear systems in both cases: nonlinear systems linearly observable and nonlinear systems with one linear unobservable mode. In the same section, we give two illustrative examples. In the last section, we give an algorithm to compute normal quadratic numbers directly and an example to check the efficiency of this algorithm.

\section{Notations and definitions}

Throughout this paper, we consider in a state neighborhood $U \subset \mathbb{R}^{n}$ of the origin, a nonlinear SISO system in the following form:

$$
\left\{\begin{array}{l}
\dot{\xi}=f(\xi)+g(\xi) u \\
y=C \xi
\end{array}\right.
$$

where, vector fields $f, g: U \subset \mathbb{R}^{n} \longrightarrow \mathbb{R}^{n}$ are assumed to be real and analytic. We assume that 0 is an equilibrium point i.e. $f(0)=0$. We set by definition $A:=\frac{\partial f}{\partial \xi}(0)$ and $B:=g(0)$. Then, system (6) can be rewritten in the following form:

$$
\left\{\begin{array}{l}
\dot{z}=A z+B u+f^{[2]}(z)+g^{[1]}(z) u+O^{3} \\
y=C z
\end{array}\right.
$$

where:

$$
\begin{aligned}
f^{[2]}(z)= & \left(\begin{array}{c}
f_{1}^{[2]}(z) \\
f_{2}^{[2]}(z) \\
\vdots \\
f_{n}^{[2]}(z)
\end{array}\right), \\
g^{[1]}(z)= & \left(\begin{array}{c}
g_{1}^{[1]}(z) \\
g_{2}^{[1]}(z) \\
\vdots \\
g_{n}^{[1]}(z)
\end{array}\right) \text { and } \\
O^{3}= & O^{3}(z, u)
\end{aligned}
$$

where for all $1 \leq i \leq n, f_{i}^{[2]}(z)$ and $g_{i}^{[1]}(z)$ are respectively homogeneous polynomials of degree 2 , respectively 1 in components of $z$.

\section{Definition 3}

i) The component $f^{[2]}(z)+g^{[1]}(z) u$ is the quadratic part of system (6).

ii) A quadratic transformation is a diffeomorphism of the form:

$$
x=z-\Phi^{[2]}(z)
$$

where $\Phi^{[2]}(z)=\left(\Phi_{1}^{[2]}(z), \ldots \ldots, \Phi_{n}^{[2]}(z)\right)^{T}$ and for all $1 \leq i \leq n, \Phi_{i}^{[2]}(z)$ is a homogeneous polynomial of degree two with respect to $z$.

Now consider the following vector spaces

$$
E_{1}=\mathbb{R}^{n}[y] \text { and } E_{2}=\mathbb{R}^{n}\left[y^{2}\right]
$$

whose elements are respectively

$$
\left(d_{1}, \ldots, d_{n}\right)^{T} y \text { and }\left(e_{1}, \ldots, e_{n}\right)^{T} y^{2}
$$

where

$$
\left(e_{1}, \ldots, e_{n}\right)^{T} \text { and }\left(d_{1}, \ldots, d_{n}\right)^{T} \in \mathbb{R}^{n} .
$$

Finally, for a fixed input $u$ we set $E=E_{2}+u E_{1}$.

For solving the problem stated above, we need the following assumption:

Assumption 4 The output is always taken equal to the first state component. Consequently, the diffeomorphism $\left(x=z-\Phi^{[2]}(z)\right)$ is such that $\Phi_{1}^{[2]}(z)=0$.

This assumption is legitimate as soon as for (1) does not fulfil Krener-Respondek conditions. However, for system which fulfils Condition 1 we do the assumption for the new output $\bar{y}=\mathfrak{F}(\mathfrak{y})$ 


\section{Definition 5}

1) Consider a second system:

$$
\left\{\begin{array}{l}
\dot{x}=A x+B u+\bar{f}^{[2]}(x)+\bar{g}^{[1]}(x) u+O^{3} \\
y=C x
\end{array} .\right.
$$

If here exists a quadratic transformation

$$
x=z-\Phi^{[2]}(z)
$$

such that, $\bar{f}^{[2]}(x)+\bar{g}^{[1]}(x) u$ equals $f^{[2]}(z)+g^{[1]}(z) u$ modulo $E$, then we say that (7) and (6) are quadratically equivalent modulo input-output injections.

2) If $\bar{f}^{[2]}(x)+\bar{g}^{[1]}(x) u$ is defined modulo an element of $E$ then we say that (6) and (7) are quadratically equivalent modulo an input-output injection.

3) If $\bar{f}^{[2]}(x)+\bar{g}^{[1]}(x) u \in E$ we say that system (6) is quadratically linearizable modulo an input-output injection.

Remark 6 If $(A, C)$ is an observable pair, then we can transform system (6) into the following form [B]:

$$
\left\{\begin{aligned}
& \dot{z}= A_{\text {obs }} z+B_{o b s} u+f^{[2]}(z)+g^{[1]}(z) u+O^{3} \\
& y=+O^{3}(z, u) \\
& \text { obs } z
\end{aligned}\right.
$$

where:

$$
\begin{aligned}
& A_{o b s}=\left(\begin{array}{ccccc}
a_{1} & 1 & 0 & \cdots & 0 \\
\vdots & 0 & \ddots & \ddots & \vdots \\
\vdots & \vdots & \ddots & \ddots & 0 \\
a_{n-1} & 0 & \cdots & 0 & 1 \\
a_{n} & 0 & \cdots & 0 & 0
\end{array}\right) \text {, } \\
& B_{\text {obs }}=\left(\begin{array}{l}
b_{1} \\
b_{2} \\
\vdots \\
\vdots \\
b_{n}
\end{array}\right) \text { and } \\
& C_{o b s}=\left(\begin{array}{llll}
1 & 0 & \ldots & 0
\end{array}\right)
\end{aligned}
$$

Remark 7 If $(A, C)$ has one unobservable real mode, then we can transform system (6) into the following form $[B]$ :

$$
\left\{\begin{aligned}
\dot{\tilde{z}}= & A_{\text {obs }} \tilde{z}+B_{o b s} u+\tilde{f}^{[2]}(z)+\tilde{g}^{[1]}(z) u \\
& +O^{3}(z, u) \\
\dot{z}_{n}= & \alpha_{n} z_{n}+\sum_{i=1}^{n-1} \alpha_{i} z_{i}+b_{n} u+f_{n}^{[2]}(z) \\
& +g_{n}^{[1]}(z) u+O^{3}(z, u) \\
y= & C_{o b s} \tilde{z}
\end{aligned}\right.
$$

where:

$$
\begin{aligned}
\tilde{z} & =\left(\begin{array}{c}
z_{1} \\
z_{2} \\
\vdots \\
\vdots \\
z_{n-1}
\end{array}\right), z=\left(\begin{array}{c}
\tilde{z} \\
z_{n}
\end{array}\right), \\
A_{\text {obs }}= & \left(\begin{array}{ccccc}
a_{1} & 1 & 0 & \ldots & 0 \\
\vdots & 0 & \ddots & \ddots & \vdots \\
\vdots & \vdots & \ddots & \ddots & 0 \\
a_{n-2} & 0 & \ldots & 0 & 1 \\
a_{n-1} & 0 & \ldots & 0 & 0
\end{array}\right), \\
B_{o b s} & =\left(\begin{array}{cccccc}
b_{1} & b_{2} & \ldots & b_{n-1}
\end{array}\right)^{T} \text { and } \\
C_{\text {obs }} & =\left(\begin{array}{lllll}
1 & 0 & \ldots & 0
\end{array}\right) .
\end{aligned}
$$

Throughout the paper, we deal with systems in forms (8) and (9).

The following gives a definition of what we will call quadratic normal forms throughout this paper.

\section{Definition 8}

1) We say that system (8) is in its quadratic normal form if:

for $1 \leq j \leq n-1$ :

$\left\{\begin{array}{l}\Delta_{f g_{j}}^{[2]}=\sum_{i=2}^{n} k_{1, i} x_{i} u \text { and } \\ \Delta_{f g_{n}}^{[2]}=\sum_{j \geq i=2}^{n} h_{i, j} x_{i} x_{j}+\sum_{i=2}^{n-1} k_{n, i} x_{i} u\end{array}\right.$

where $\Delta_{f g_{j}}^{[2]}=f_{j}^{[2]}(z)+g_{j}^{[1]}(z) u$.

Coefficients: $h_{i, j}$ for $2 \leq i \leq j \leq n$ and $k_{n, i}$ for $2 \leq i \leq n-1$ are called normal quadratic numbers.

2) Through an abuse of language ${ }^{1}$, we say that system (9) is in its normal form if:

for $1 \leq j \leq n-2$, :

$$
\left\{\begin{aligned}
\Delta_{f g_{j}}^{[2]}= & \sum_{i=2}^{n} k_{1, i} x_{i} u \text { and } \\
\Delta_{f g_{n-1}}^{[2]}= & \sum_{j \geq i=2}^{n} h_{i, j} x_{i} x_{j}+h_{1, n} x_{1} x_{n} \\
& +\sum_{i=2}^{n} k_{n-1, i} x_{i} u, \\
\Delta_{f g_{n}}^{[2]}= & \sum_{j \geq i=2}^{n} l_{i, j} x_{i} x_{j}+\sum_{j=2}^{n} l_{1, j} x_{1} x \\
& +\sum_{i=2}^{n} k_{n, i} x_{i} u
\end{aligned}\right.
$$

Coefficients: $h_{i, j}$ for $2 \leq i \leq j \leq n$ and $k_{n-1, i}$ for $2 \leq i \leq n-1$ are called normal quadratic numbers.

Throughout this work we will use matrix calculus to compute normal quadratic numbers, for this reason we adopt the following notations:

\footnotetext{
${ }^{1}$ The linear unobservable part has not yet been reduced.
} 


$$
\Phi_{i}^{[2]}(z)=z^{T} \Phi_{i} z \text { and } f_{i}^{[2]}(z)=z^{T} f_{i} z
$$

where for all $1 \leq i \leq n, \Phi_{i}$ and $f_{i}$ are symmetric matrices. We make the notational $\Phi_{i}^{[2]}(z)$ and $f_{i}^{[2]}(z)$ for all $1 \leq i \leq n$ of indicating quadratic functions and the notational $\Phi_{i}$ and $f_{i}$ of indicating the corresponding matrices. In the same way, for all $1 \leq i \leq n$, the $1 \times n$ vectors $g_{i}=\left(g_{i, 1}, \ldots, g_{i, n}\right)$ are associated with functions $g_{i}^{[1]}(z)$ i.e. $g_{i}^{[1]}(z)=z^{T} g_{i}=g_{i}^{T} z$. With those notations, we have:

$$
\begin{aligned}
& \Phi=\left(\Phi_{1}, \ldots ., \Phi_{n}\right)^{T}, f=\left(f_{1}, \ldots, f_{n}\right)^{T} \\
& \text { and } g=\left(g_{1}, \ldots ., g_{n}\right)^{T} .
\end{aligned}
$$

\section{Quadratic Normal Forms}

In this section we will state two theorems concerning the setting of systems (8) and (9) in their normal forms (10) and (11) respectively. Each theorem is followed by an illustrative example. The end of this section is devoted to the necessary and sufficient conditions to cancel the quadratic terms in the last dynamic of normal form (11). With these intentions, we will start by giving the necessary and sufficient algebraic conditions under which two systems in the form of (6) are quadratically equivalent modulo an input-output injection.

Proposition 9 System (6) is quadratically equivalent modulo an input-output injection to system (7), if and only if the following two homological equations are satisfied:

i) $\quad A \Phi^{[2]}(z)-\frac{\partial \Phi^{[2]}}{\partial z} A z=\bar{f}^{[2]}(z)-f^{[2]}(z)$ modulo $E_{2}$

ii) $\quad-\frac{\partial \Phi^{[2]}}{\partial z} B=\bar{g}^{[1]}(z)-g^{[1]}(z)$ modulo $E_{1}$

where $\frac{\partial \Phi^{[2]}}{\partial z} A z:=\left(\frac{\partial \Phi_{1}^{[2]}(z)}{\partial z} A z, \ldots \ldots, \frac{\partial \Phi_{n}^{[2]}(z)}{\partial z} A z\right)^{T}$ and for all $1 \leq i \leq n \frac{\partial \Phi_{i}^{[2]}(z)}{\partial z}$ is the Jacobian matrix of $\Phi_{i}^{[2]}(z)$.

Now we will express these homological equations in terms of matrices, this allows us to compute in section 4 normal quadratic numbers. For this, we set by definition

$$
\left\{\begin{array}{c}
A^{T} \Phi:=\left(A^{T} \Phi_{1}, \ldots ., A^{T} \Phi_{n}\right) \\
B^{T} \Phi:=\left(B^{T} \Phi_{1}, \ldots ., B^{T} \Phi_{n}\right) \\
\text { and } \Phi A:=\left(\Phi_{1} A, \ldots . ., \Phi_{n} A\right)
\end{array}\right.
$$

Let $\beta=\left(\beta_{1}, \ldots ., \beta_{n}\right)^{T}$ and $\gamma=\left(\gamma_{1}, \ldots ., \gamma_{n}\right)^{T}$ where for all $1 \leq i \leq n$, we shall frequently make the notational abuse

$$
\begin{aligned}
& \beta_{i}:=\left(\begin{array}{cccc}
\beta_{1, i} & 0 & \ldots & 0 \\
0 & 0 & \ddots & 0 \\
\vdots & \vdots & \ddots & \vdots \\
0 & 0 & \ldots & 0
\end{array}\right) \text { and } \\
& \gamma_{i}:=\left(\gamma_{1, i}, 0, \ldots ., 0,0\right)
\end{aligned}
$$

where $\beta_{i}$ and $\gamma_{i} \in \mathbb{R}$. With this notation we have: $\beta_{1, i} y^{2}=z^{T} \beta_{i} z \in E_{2}$ and $\gamma_{1, i} y=\gamma_{i} z \in E_{1}$.

The next result gives the matrix version of Proposition 9 .

Corollary 10 System (6) is quadratically equivalent modulo an output-input-injection to system ( 7$)$, if and only if there exist $\beta$ and $\gamma$ such that:

$$
\begin{aligned}
\bar{\Phi}-\left(A^{T} \Phi+\Phi A\right) & =\bar{f}-f+\beta \\
-2 B^{T} \Phi & =\bar{g}-g+\gamma
\end{aligned}
$$

where

i) in the observable case

$$
\bar{\Phi}=\left(\Phi_{2}, \ldots ., \Phi_{n}, 0\right)
$$

ii) in the unobservable case

$$
\bar{\Phi}=\left(\Phi_{2}, \ldots ., \Phi_{n-1}, 0, \bar{\Phi}_{n}\right)
$$

with $\bar{\Phi}_{n}=\sum_{i=2}^{n} \alpha_{i} \Phi_{i}$

Proof. Consider the homological equations:

i) $\quad \bar{f}^{[2]}(z)-f^{[2]}(z)+\beta^{[2]}\left(z_{1}\right)=A \Phi^{[2]}(z)-\frac{\partial \Phi^{[2]}}{\partial z} A z$

ii) $\bar{g}^{[1]}(z)-g^{[1]}(z)+\gamma^{[1]}\left(z_{1}\right)=-\frac{\partial \Phi^{[2]}}{\partial z} B$

Using matrix notations:

$$
\begin{aligned}
& \Phi^{[2]}(z)=\left(z^{T} \Phi_{1} z, \ldots, z^{T} \Phi_{n} z\right)^{T} \text { and } \\
& \Phi^{[2]}(z)=A \Phi^{[2]}(z) .
\end{aligned}
$$

i) As in the linearly observable case

$$
A=\left(\begin{array}{ccccc}
a_{1} & 1 & 0 & \cdots & 0 \\
\vdots & 0 & \ddots & \ddots & \vdots \\
\vdots & \vdots & \ddots & \ddots & 0 \\
a_{n-1} & 0 & \cdots & 0 & 1 \\
a_{n} & 0 & \cdots & 0 & 0
\end{array}\right)
$$


then $\bar{\Phi}^{[2]}(z)=\left(\Phi_{2}^{[2]}(z), \ldots ., \Phi_{n}^{[2]}(z), 0\right)$ because $\Phi_{1}^{[2]}(z)=0$.

ii) For the unobservable case

$$
A=\left(\begin{array}{ccccc}
a_{1} & 1 & 0 & \cdots & 0 \\
\vdots & 0 & \ddots & \ddots & \vdots \\
\vdots & \vdots & \ddots & \ddots & 0 \\
a_{n-2} & 0 & \cdots & 0 & 1 \\
a_{n-1} & 0 & \cdots & 0 & 0 \\
a_{1} & \alpha_{2} & \cdots & \cdots & \alpha_{n}
\end{array}\right)
$$

we obtain

$$
\bar{\Phi}(z)=\left(\Phi_{2}^{[2]}(z), \ldots . ., \Phi_{n-1}^{[2]}(z), 0, \bar{\Phi}_{n}^{[2]}(z)\right)
$$

where

$$
\bar{\Phi}_{n}^{[2]}=\sum_{i=2}^{n} \alpha_{i} \Phi_{i}^{[2]}
$$

Now,

$$
\frac{\partial \Phi^{[2]}(z)}{\partial z} A z=\left(\frac{\partial \Phi_{1}^{[2]}(z)}{\partial z} A z, \ldots, \frac{\partial \Phi_{n}^{[2]}(z)}{\partial z} A z\right)^{T}
$$

as for all $i \in[1, n]$;

$$
\Phi_{i}^{[2]}(z)=z^{T} \Phi_{i} z
$$

then

$$
\frac{\partial \Phi^{[2]}(z)}{\partial z} A z=\left(\begin{array}{c}
z^{T} A^{T} \Phi_{1} z+z^{T} \Phi_{1} A z \\
\vdots \\
z^{T} A^{T} \Phi_{n} z+z^{T} \Phi_{n} A z
\end{array}\right)^{T}
$$

which by definition is:

$$
\frac{\partial \Phi^{[2]}(z)}{\partial z} A z=z^{T}\left(A^{T} \Phi+\Phi A\right) z
$$

In the same way we have

$$
\frac{\partial \Phi_{i}^{[2]}(z)}{\partial z} B=B^{T} \Phi_{i} z+z^{T} \Phi_{i} B=2 B^{T} \Phi_{i} z
$$

because $\Phi_{i}$ is symmetric. Using previous results, in homological equations of Proposition 9, we obtain equations stated in Corollary 10:

$$
\begin{aligned}
& \bar{f}-f+\beta=\bar{\Phi}-\left(A^{T} \Phi+\Phi A\right) \\
& \bar{g}-g+\gamma=-2 B^{T} \Phi
\end{aligned}
$$

\subsection{Nonlinear systems linearly ob- servable case}

In this subsection, we will compute quadratic normal form for linearly observable systems. It is clear from the structure of the quadratic normal form (10) that a system is quadratically linearizable if and only if all its normal quadratic numbers are equal to zero. Thus, a system in the form (8) is quadratically linearizable if and only if its quadratic part satisfies the homological equations of Corollary 10 where $\bar{f}=0$ and $\bar{g}=0$. Now, under the Assumption 4 and thanks to the structure of matrix $A_{o b s}$, homological equations for a quadratically linearizable system which is linearly observable are:

$$
\begin{aligned}
& \begin{cases}\text { (a.1) } & \text { for } i \in[2, n] \\
& \Phi_{i}=\left(A_{o b s}^{T} \Phi_{i-1}+\Phi_{i-1} A_{o b s}\right)+\Delta_{f_{i-1}} \\
\text { (a.2) } & 0=\left(A_{o b s}^{T} \Phi_{n}+\Phi_{n} A_{o b s}\right)+\Delta_{f_{n}}\end{cases} \\
& \text { and } \\
& \begin{cases}(b .1) & -g_{1}+\gamma_{1}=0 \\
\text { (b.2) } & \text { for } i \in[2, n], 2 B^{T} \Phi_{i}+\Delta_{g_{i}}=0\end{cases}
\end{aligned}
$$

where $\Delta_{f_{j}}=-f_{j}+\beta_{j}$ and $\Delta_{g_{j}}=-g_{j}+\gamma_{j}$.

Equation (a.1) gives explicitly $\Phi_{i}$ for all $2 \leq i \leq n$. Then all quadratic terms in the $(n-1)$ first dynamics are deleted.

Remark 11 Moreover, equation (a.2) is equivalent to the following equation:

$$
\sum_{s=0}^{n-1}\left(\sum_{j=0}^{s} C_{j}^{s}\left(A^{T}\right)^{s-j}\left(\Delta_{f_{n-s}}\right) A^{j}\right)=0
$$

and equations (b.1) and (b.2) are re-written as follows:

$$
\left\{\begin{array}{l}
\text { (b.1) }-g_{1}+\gamma_{1}=0 \\
\text { (b.2) for } i \in[2, n] \\
2 B^{T} \sum_{k=0}^{i-2} \sum_{j=0}^{k} C_{j}^{k}\left(A^{T}\right)^{k-j} \Delta_{f_{i-1-k}} A^{j}+\Delta_{g_{i}}=0
\end{array}\right.
$$

Under Assumption 4, using Proposition 9, we obtain the following theorem which gives normal forms for nonlinear linearly observable systems.

Theorem 12 There exists a quadratic diffeomorphism which transforms the quadratic part of system (8) into the quadratic normal form (10) modulo E.

Let us consider an example to illustrate the above theorem: 
Example 13 Consider the following system:

$$
\left\{\begin{array}{l}
\dot{z}_{1}=a_{1} z_{1}+z_{2}+k_{1} z_{2}^{2}+l_{1} z_{3}^{2} \\
\dot{z}_{2}=a_{2} z_{1}+z_{3}+k_{2} z_{2}^{2}+l_{2} z_{3}^{2} \\
\dot{z}_{3}=a_{3} z_{1}+k_{3} z_{2}^{2}+l_{3} z_{3}^{2} \\
y=z_{1} .
\end{array}\right.
$$

Homological equations of Proposition 9 for system (12) are:

$$
\left\{\begin{aligned}
\Phi_{1}^{[2]}(z)= & 0 \\
\Phi_{2}^{[2]}(z)= & -k_{1} z_{2}^{2}-l_{1} z_{3}^{2}+\beta_{1,1} z_{1}^{2} \\
\Phi_{3}^{[2]}(z)= & 2 \beta_{1,1} z_{1}\left(a_{1} z_{1}+z_{2}\right)-2 k_{1} z_{2}\left(a_{2} z_{1}+z_{3}\right) \\
& -2 l_{1} a_{3} z_{1} z_{3}-k_{2} z_{2}^{2}-l_{2} z_{3}^{2}+\beta_{1,2} z_{1}^{2}
\end{aligned}\right.
$$

then by choosing:

$$
\begin{aligned}
& \beta_{1,1}=l_{1} a_{3} a_{1}+2 k_{1} a_{2}+l_{2} a_{3} \\
& \text { and } \\
& \beta_{1,2}=-3 \beta_{1,1} a_{1}+k_{1} a_{2} a_{1}+k_{2} a_{2}+k_{1} a_{3},
\end{aligned}
$$

the quadratic normal form of system (12) is:

$$
\left\{\begin{aligned}
\dot{x}_{1}= & a_{1} x_{1}+x_{2}+\beta_{1,1} x_{1}^{2}+O^{3} \\
\dot{x}_{2}= & a_{2} x_{1}+x_{3}+\beta_{1,2} x_{1}^{2}+O^{3} \\
\dot{x}_{3}= & a_{3} x_{1}+\left(k_{3}-2 \beta_{1,1}+2 k_{1} a_{2}\right) x_{2}^{2}+\beta_{1,3} x_{1}^{2} \\
& +\left(2 l_{1} a_{3}+2 k_{2}\right) x_{2} x_{3}+\left(l_{3}+2 k_{1}\right) x_{3}^{2}+O^{3} \\
y= & x_{1} .
\end{aligned}\right.
$$

\subsection{Nonlinear systems with one linear unobservable mode case}

In the same way as in the above subsection, under Assumption 4 and Proposition 9 we obtain the following result.

Theorem 14 There is a quadratic transformation which transforms the quadratic part of system (9) in to the quadratic normal form (11).

Now we are ready to highlight the the usefulness of our normal form to analyze the observability.

\section{Remark 15}

1) We call the unobservability submanifold the subset $S_{n-1}$ of $U$ given by:

$$
S_{n-1}=\left\{\begin{array}{l}
x \in U, \text { such that } \\
\sum_{i=1}^{n-1} h_{i, n} x_{i}+2 h_{n, n} x_{n}+k_{n-1, n} u=0
\end{array}\right\}
$$

Thus, for a fixed input $u$, when system evolves on $S_{n-1}$ we lose the linear and quadratic observability.

2) If $x \in S_{n-1}$ and $k_{n-1, n} \neq 0$, then with an appropriate change of input u (universal input [GB]), we can modify $S_{n-1}$ to locally restore the quadratic observability. Moreover if $k_{n-1, n}=0$ and if there exists $i \in[1, n]$ such that $k_{i, n} \neq 0$, then quadratic observability is restored.

3) If $x \in S_{n-1}$ such that $u$ is not a function of $x_{n}$ and for all $i \in[1, n]$ we have $k_{i, n}=0$, then we use coefficient $\alpha_{n}$ to study the detectability propriety. For this we distinguish three cases:

a) if $\alpha_{n}<0$ then the state $x_{n}$ is detectable,

b) if $\alpha_{n}>0$ then $x_{n}$ is unstable and consequently undetectable,

c) if $\alpha_{n}=0$ we can use the center manifold theory in order to analyze stability or instability of $x_{n}$ and consequently its detectability or undetectability.

Remark 16 Let $\Phi^{[2]}(x)=\left(0, \ldots ., \Phi_{n}^{[2]}(x)\right)$ be the quadratic part of the diffeomorphism which gives the above theorem. The diffeomorphism $\Phi_{2}^{[2]}(x)$ is well determined from $f_{1}^{[2]}(x)$ and for all $3 \leq i \leq n-1$, $\Phi_{i}^{[2]}(x)$ is determined from $f_{i}^{[2]}(x)$ and the derivatives of $\Phi_{j}^{[2]}(x)$ and $f_{j}^{[2]}(x)$ for $2 \leq j \leq i-1$. However, the choice of $\Phi_{n}^{[2]}(x)$ is free. So it is interesting to use $\Phi_{n}^{[2]}(x)$ to cancel quadratic terms in the last $\dot{x}_{n}$ dynamic. For this $\Phi_{n}^{[2]}(x)$ must fulfil the following equation:

$$
\begin{aligned}
& \alpha_{n} \Phi_{n}^{[2]}(x)+\sum_{i=1}^{n-1} \alpha_{i} \Phi_{i}^{[2]}(x)=\frac{\partial \Phi_{n}^{[2]}}{\partial x} A_{o b s} x+\Delta_{f_{n}}^{[2]} \\
& \text { where } \Delta_{f_{j}}^{[2]}=-f_{j}^{[2]}(x)+\beta_{j}^{[2]}\left(x_{1}\right) .
\end{aligned}
$$

Unfortunately, the above equation is not fulfilled for arbitrary $\alpha_{n}$ and $\left(a_{i}\right)_{1 \leq i \leq n}$, as we will show in the next example.

Example 17 Consider the following system:

$$
\left\{\begin{aligned}
\dot{z}_{1}= & a_{1} z_{1}+z_{2}+k_{1} z_{2}^{2}+l_{1} z_{3}^{2} \\
\dot{z}_{2}= & a_{2} z_{1}+k_{2} z_{2}^{2}+l_{2} z_{3}^{2} \\
\dot{z}_{3}= & \alpha_{1} z_{1}+\alpha_{2} z_{2}+\alpha_{3} z_{3}+k_{3} z_{2}^{2} \\
& +l_{3} z_{3}^{2} \\
y= & z_{1}
\end{aligned}\right.
$$

The homological equations of Proposition 9 for system (14) are:

$$
\left\{\begin{array}{l}
\Phi_{1}^{[2]}(z)=0 \\
\Phi_{2}^{[2]}(z)=-k_{1} z_{2}^{2}-l_{1} z_{3}^{2}+\beta_{1,1} z_{1}^{2}
\end{array}\right.
$$

rewriting $\Phi_{3}^{[2]}(z)=z^{T} \Phi_{3} z$ where:

$$
\Phi_{3}=\left(\begin{array}{lll}
\phi_{1,1} & \phi_{1,2} & \phi_{1,3} \\
\phi_{1,2} & \phi_{2,2} & \phi_{2,3} \\
\phi_{1,3} & \phi_{2,3} & \phi_{3,3}
\end{array}\right)
$$


then, if $\alpha_{3} \neq 0$ and $a_{2} \neq 0$ we obtain:

$$
\left(\begin{array}{l}
\phi_{1,3} \\
\phi_{2,3} \\
\phi_{3,3}
\end{array}\right)=\left(\begin{array}{c}
\alpha_{2} \frac{l_{3}+\alpha_{1} l_{1}}{\alpha_{3}} \\
\frac{l_{3}+\alpha_{1} l_{1}}{\alpha_{3} a_{2}}\left(-a_{1} \alpha_{2}-\alpha_{1}\right) \\
-\frac{l_{3}+\alpha_{1} l_{1}}{\alpha_{3}}
\end{array}\right) .
$$

If $\alpha_{3} \neq 0, a_{2} \neq 0$ and $\left(\alpha_{3}-a_{1}\right)\left(\alpha_{3}^{2}-2 \alpha_{3} a_{1}-4 a_{2}\right) \neq 0$, then:

$$
\begin{aligned}
\left(\begin{array}{l}
\phi_{1,1} \\
\phi_{1,2} \\
\phi_{2,2}
\end{array}\right)= & \left(\begin{array}{ccc}
\left(\alpha_{3}-2 a_{1}\right) & -2 a_{2} & 0 \\
-1 & \left(\alpha_{3}-a_{1}\right) & -a_{2} \\
0 & -2 & \alpha_{3}
\end{array}\right)^{-1} \times \\
& \left(\begin{array}{l}
2 \alpha_{1} \phi_{1,3}-\alpha_{2} \beta_{1,1}+\beta_{1,2} \\
\alpha_{2} \phi_{1,3}+\alpha_{1} \phi_{2,3} \\
2 \alpha_{2} \phi_{2,3}+\alpha_{2} k_{1}+k_{3}
\end{array}\right)
\end{aligned}
$$

and $\Phi_{3}^{[2]}(z)$ cancels all quadratic terms in $\dot{z}_{3}$. Finally, choosing $\beta_{1,1}=k_{1} a_{2}$ we obtain:

$$
\left\{\begin{aligned}
\dot{x}_{1}= & a_{1} x_{1}+x_{2}+\beta_{1,1} x_{1}^{2}+O^{3} \\
\dot{x}_{2}= & a_{2} x_{1}+k_{2} x_{2}^{2}+\left(2 l_{1} \alpha_{3}+l_{2}\right) x_{3}^{2}+2 l_{1} \alpha_{1} x_{1} x_{3} \\
& +2 l_{1} \alpha_{2} x_{2} x_{3}+\left(-2 a_{1} \beta_{1,1}+\beta_{1,2}\right) x_{1}^{2}+O^{3} \\
\dot{x}_{3}= & \alpha_{1} x_{1}+\alpha_{2} x_{2}+\alpha_{3} x_{3}+O^{3} \\
y= & x_{1}
\end{aligned}\right.
$$

Thus, for $\alpha_{3} \neq 0, a_{2} \neq 0$ and

$$
\left(\alpha_{3}-a_{1}\right)\left(\alpha_{3}^{2}-2 \alpha_{3} a_{1}-4 a_{2}\right) \neq 0
$$

there is $\Phi_{3}^{[2]}(x)$ which fulfils (13).

\section{Computation algorithm of normal quadratic numbers}

In this section, we give an easily implementable algorithm in order to compute normal quadratic numbers of quadratic normal forms (10) and (11). This allows us to define the observability quadratic equivalence class of a system without formally solving homological equations 9. For this, we introduce what we call characteristic matrices, of which some coefficients are the normal quadratic numbers. We consider again Example 13 to show the power of this algorithm. Moreover, for the linearly unobservable case we point out normal quadratic numbers which allow us to recover, at least locally, the quadratic observability, and so to be able to design an observer. At the end of this section, we highlight our purpose by the bio-reactor example.

\subsection{Nonlinear systems linearly ob- servable case}

The next theorem explicitly gives the matrix family $\left(\beta_{i}\right)_{1 \leq i \leq n}$ and $\left(\Phi_{i}\right)_{1 \leq i \leq n}$ which transforms the system into its quadratic normal form.

\section{Theorem 18}

i) The family $\left(\beta_{i}\right)_{1 \leq i \leq n}$ viewed as real numbers, is the solution of the following algebraic linear system:

$$
D\left(\begin{array}{c}
\beta_{1,1} \\
\beta_{1,2} \\
\vdots \\
\vdots \\
\beta_{1, n}
\end{array}\right)=(C F)^{T}
$$

where

$$
\begin{aligned}
D & =\left(\begin{array}{ccccc}
d_{1,1} & \cdots & \cdots & d_{1, n-1} & 1 \\
\vdots & . & \cdot & 1 & 0 \\
\vdots & . & . & 0 & 0 \\
d_{n-1,1} & 1 & 0 & 0 & 0 \\
1 & 0 & 0 & 0 & 0
\end{array}\right), \\
F & :=\sum_{s=0}^{n-1}\left(\sum_{j=0}^{s} C_{j}^{s}\left(A^{T}\right)^{s-j} f_{n-s} A^{j}\right) \text { and } \\
C & =(1,0 \ldots, 0),
\end{aligned}
$$

and for all $1 \leq k \leq n$ we have

$$
\begin{aligned}
\left(\begin{array}{c}
d_{1, k} \\
d_{2, k} \\
\vdots \\
1 \\
0 \\
\vdots \\
0
\end{array}\right)= & \left(\begin{array}{c}
d_{1, k} \\
d_{2, k} \\
\vdots \\
d_{n-(k-1)}, k \\
0 \\
\vdots \\
0
\end{array}\right) \\
& =\sum_{j=0}^{n-k} C_{j}^{n-k}\left(A^{T}\right)^{j} L A^{n-k-j} C^{T}
\end{aligned}
$$

where

$$
L=\left(\begin{array}{cccc}
1 & 0 & \ldots & 0 \\
0 & 0 & \ddots & 0 \\
\vdots & \vdots & \ddots & \vdots \\
0 & 0 & \ldots & 0
\end{array}\right)
$$

ii) For $2 \leq i \leq n$, matrices $\Phi_{i}$ which fulfilled equation (a.1) are:

$$
\Phi_{i}=\sum_{k=0}^{i-2} \sum_{j=0}^{k} C_{j}^{k}\left(A^{T}\right)^{k-j}\left(\Delta_{f_{i-1-k}}\right) A^{j}
$$

Remark 19 If matrix family $\left(\beta_{i}\right)_{1 \leq i \leq n}$ and $\left(\Phi_{i}\right)_{1 \leq i \leq n}$ which are given in Theorem 18 below fulfil equations (a.2), (b.1) and (b.2) then system (8) is fully quadratically linearizable. However, in general these equations do not admit solutions. We use their left members to give the following definition: 
Definition 20 We call characteristic matrices associated with system (8) the following matrices:

$$
\begin{aligned}
& M= \sum_{s=0}^{n-1}\left(\sum_{j=0}^{s} C_{j}^{s}\left(A^{T}\right)^{s-j}\left(\Delta_{f_{n-s}}\right) A^{j}\right) \\
& N_{1}=-g_{1}+\gamma_{1}, \\
& \text { and for } i \in[2, n] \\
& N_{i}=2 B^{T} \sum_{k=0}^{i-2} \sum_{j=0}^{k} C_{j}^{k}\left(A^{T}\right)^{k-j} \Delta_{f_{i-1-k}} A^{j} \\
& \quad+\Delta_{g_{i}}
\end{aligned}
$$

We call characteristic numbers; coefficients of characteristic matrices which are not generically equal to zero.

Now we can state the link between normal quadratic numbers and elements of characteristic matrices.

We know from Remark 19 that $M=0$ and $N_{l}=0$ for $1 \leq l \leq n$ are the conditions of the full quadratic linearization, thus we have:

Proposition 21 Quadratic normal numbers of normal form (10) are given by:

$\forall i, j \in[2, n]$ and $l \in[2, n]$

$$
\left\{\begin{aligned}
h_{i, j} & =-2 M_{i, j} \text { for } i \neq j \\
h_{i, i} & =-M_{i, i} \\
k_{1, j} & =-N_{1, j} \\
k_{l, j} & =-N_{l, j}
\end{aligned}\right.
$$

where $M_{i, j}$ is the $i^{\text {th }}$ row and the $j^{\text {th }}$ column element of characteristic matrix $M$, and $N_{l, j}$ is the $j^{\text {th }}$ element of the row vector $N_{l}$.

\section{Remark 22}

1) The fact that $M_{1, j}=0$ for all $1 \leq j \leq n$ is equivalent to the algebraic linear system (15).

2) In the normal form (10) we have at most $\frac{n(n-1)}{2}$ normal quadratic numbers $x_{i} x_{j}$ and $n(n-1)$ normal quadratic numbers $x_{i} u$. In fact, we have

i) $n \frac{n(n+1)}{2}$ constraints given by the $n$ symmetric matrices $\left(f_{i}\right)_{1 \leq i \leq n}$,

ii) $n^{2}$ constraints given by the $n$ matrices $\left(g_{i}\right)_{1 \leq i \leq n}$,

iii) $(n-1) \frac{n(n+1)}{2}$ symmetric matrices $\phi_{i} \quad 2 \leq i \leq$ $n$ which are completely given by the $(n-1)$ first $d y$ namics and

iv) $n$ degrees of freedom given by output injections $\left(\beta_{i}\right)_{1 \leq i \leq n}$,

v) $n$ degrees of freedom given by output injections $\left(\gamma_{i}\right)_{1 \leq i \leq n}$.

Thus, the number of normal quadratic numbers is:

$n \frac{n(n+1)}{2}-(n-1) \frac{n(n+1)}{2}-2 n+n^{2}=\frac{3}{2} n(n-1)$.
Example 23 Let us consider again (12) to highlight the power of the characteristic matrices to compute quadratic normal forms.

$$
\left\{\begin{array}{l}
\dot{z}_{1}=a_{1} z_{1}+z_{2}+k_{1} z_{2}^{2}+l_{1} z_{3}^{2} \\
\dot{z}_{2}=a_{2} z_{1}+z_{3}+k_{2} z_{2}^{2}+l_{2} z_{3}^{2} \\
\dot{z}_{3}=a_{3} z_{1}+k_{3} z_{2}^{2}+l_{3} z_{3}^{2}
\end{array}\right.
$$

From Theorem 18, we obtain:

$$
\left\{\begin{aligned}
\beta_{1,1}= & l_{1} a_{3} a_{1}+2 k_{1} a_{2}+l_{2} a_{3} \\
\beta_{1,2}= & -3 \beta_{1,1} a_{1}+k_{1} a_{2} a_{1}+k_{2} a_{2}+k_{1} a_{3} \\
\beta_{1,3}= & -3 a_{1}^{2} \beta_{1,1}-\beta_{1,1} a_{2}-2 a_{1} \beta_{1,2} \\
& -\left(a_{1}^{2}+a_{2}\right) \beta_{1,1}+2 a_{2}^{2} k_{1}+2 a_{3}^{2} l_{1}
\end{aligned}\right.
$$

then

$$
\left\{\begin{array}{ll}
M_{1, j} & =0 \text { for all } 1 \leq j \leq 3 \\
M_{2,2} & =-2 a_{2} k_{1}-k_{3}+2 \beta_{1,1} \\
M_{2,3}=M_{3,2} & =-a_{3} l_{1}-k_{2} \\
M_{3,3} & =-l_{3}-2 k_{1}
\end{array} .\right.
$$

By Proposition 21 the normal quadratic numbers are:

$$
\left\{\begin{array}{l}
h_{1,1}=h_{1,2}=h_{1,3}=0 \\
h_{2,2}=2 a_{2} k_{1}+k_{3}-2 \beta_{1,1} \\
h_{2,3}=2 a_{3} l_{1}+2 k_{2} \\
h_{3,3}=l_{3}+2 k_{1}
\end{array}\right.
$$

So, the quadratic normal form associated to (18) is:

$$
\left\{\begin{aligned}
\dot{x}_{1} & =a_{1} x_{1}+x_{2}+\beta_{1,1} x_{1}^{2}+O^{3} \\
\dot{x}_{2} & =a_{2} x_{1}+x_{3}+\beta_{1,2} x_{1}^{2}+O^{3} \\
\dot{x}_{3} & =a_{3} x_{1}+\left(k_{3}-2 \beta_{1,1}+2 k_{1} a_{2}\right) x_{2}^{2} \\
& +\left(l_{3}+2 k_{1}\right) x_{3}^{2}+\left(2 l_{1} a_{3}+2 k_{2}\right) x_{2} x_{3}+O^{3} .
\end{aligned}\right.
$$

Conditions of the full quadratic linearization are $M_{i, j}=0$ for all $2 \leq i \leq j \leq 3$.

\subsection{Nonlinear systems with one linear unobservable mode case}

In this subsection, as previously for the linear observable case, firstly we introduce and compute characteristic matrices of (11), we deduce normal quadratic numbers and finally we discuss the quadratic observability and stability properties. For this, setting $\bar{f}=0$ and $\bar{g}=0$ in homological equations of Corollary 10, we obtain:

$$
\begin{aligned}
\Phi_{i} & =\underbrace{\left(A^{T} \Phi_{i-1}+\Phi_{i-1} A\right)+\Delta_{f_{i-1}}}_{\text {and for } i \in[2, n-2]} \\
0 & =\left(A^{T} \Phi_{n-1}+\Phi_{n-1} A\right)+\Delta_{f_{n-1}} \\
& =\sum_{s=0}^{n-2} \sum_{j=0}^{s} C_{j}^{s}\left(A^{T}\right)^{s-j}\left(\Delta_{f_{n-s}}\right) A^{j} \\
\alpha_{n} \Phi_{n} & =-\sum_{i=1}^{n-1} \alpha_{i} \Phi_{i}+\left(A^{T} \Phi_{n}+\Phi_{n} A\right)+\Delta_{f_{n}}
\end{aligned}
$$

The following is corresponding result to Theorem 18 for linearly unobservable case. 


\section{Theorem 24}

i) The family $\left(\beta_{i}\right)_{1 \leq i \leq n-1}$ viewed as real numbers, is the solution of the following algebraic linear system:

$$
\bar{D}\left(\begin{array}{c}
\beta_{1,1} \\
\beta_{1,2} \\
\vdots \\
\vdots \\
\beta_{1, n-1}
\end{array}\right)=(C F)^{T}
$$

where

$$
\begin{aligned}
\bar{D} & =\left(\begin{array}{ccccc}
d_{1,1} & \cdots & \cdots & d_{1, n-1} & 1 \\
\vdots & \cdot & \cdot & 1 & 0 \\
\vdots & \cdot & \cdot & 0 & 0 \\
d_{n-1,1} & 1 & 0 & 0 & 0 \\
1 & 0 & 0 & 0 & 0
\end{array}\right) \\
F & :=\sum_{s=0}^{n-2}\left(\sum_{j=0}^{s} C_{j}^{s}\left(A^{T}\right)^{s-j} f_{n-1-s} A^{j}\right) \text { and } \\
C & =(1,0 \ldots, 0),
\end{aligned}
$$

and for all $1 \leq k \leq n-1$, we have

$$
\begin{aligned}
\left(\begin{array}{c}
d_{1, k} \\
d_{2, k} \\
\vdots \\
1 \\
0 \\
0
\end{array}\right) & =\left(\begin{array}{c}
d_{1, k} \\
d_{2, k} \\
\vdots \\
d_{n-1-(k-1)}, k \\
0 \\
0
\end{array}\right) \\
& =\sum_{j=0}^{n-1-k} C_{j}^{n-1-k}\left(A^{T}\right)^{j} t A^{n-1-k-j} C^{T}
\end{aligned}
$$

where

$$
E=\left(\begin{array}{cccc}
1 & 0 & \ldots & 0 \\
0 & 0 & \ddots & 0 \\
\vdots & \vdots & \ddots & \vdots \\
0 & 0 & \ldots & 0
\end{array}\right)
$$

ii) For $2 \leq i \leq n-1$, matrices $\Phi_{i}$ which satisfy equation (a.1) are:

$$
\Phi_{i}=\sum_{k=0}^{i-2} \sum_{j=0}^{k} C_{j}^{k}\left(A^{T}\right)^{k-j}\left(\Delta_{f_{i-1-k}}\right) A^{j}
$$

Remark 25 If $\Phi_{n-1}$ satisfies the equation (a.2) i.e.

$$
\sum_{s=1}^{n-2} \sum_{j=0}^{s-1} C_{j}^{s-1}\left(A^{T}\right)^{s-j}\left(\Delta_{f_{n-s}}\right) A^{j+1}+\Delta_{f_{n-1}}=0
$$

and $\Phi_{n}$ satisfies the following equation

$$
\alpha_{n} \Phi_{n}=-\sum_{i=1}^{n-1} \alpha_{i} \Phi_{i}+\left(A^{T} \Phi_{n}+\Phi_{n} A\right)+\Delta_{f_{n}}
$$

and if

$$
\begin{aligned}
& \text { (b.1) }-g_{1}+\gamma_{1}=0 \\
& \text { (b.2) and for } i \in[2, n] \\
& 2 B^{T} \sum_{k=0}^{i-2} \sum_{j=0}^{k} C_{j}^{k}\left(A^{T}\right)^{k-j} \Delta_{f_{i-1-k}} A^{j}+\Delta_{g_{i}}=0
\end{aligned}
$$

then system (9) is fully quadratically linearizable.

Definition 26 Characteristic matrices of the observable part of (9) are given by:

$$
\begin{aligned}
& M=\sum_{s=0}^{n-2} \sum_{j=0}^{s} C_{j}^{s}\left(A^{T}\right)^{s-j}\left(\Delta_{f_{n-1-s}}\right) A^{j}, \\
& N_{1}=-g_{1}+\gamma_{1} \\
& \text { and for } i \in[2, n-1] \\
& N_{i}=2 B^{T} \sum_{k=0}^{i-2} \sum_{j=0}^{k} C_{j}^{k}\left(A^{T}\right)^{k-j} \Delta_{f_{i-1-k}} A^{j}+\Delta_{g_{i}}
\end{aligned}
$$

Now, from the previous definition we present relations between normal quadratic numbers of (11) and elements of its characteristic matrices.

Proposition 27 Quadratic normal numbers associated with the observable part of normal form (11) are:

$\forall i, j \in[2, n]$ and $l \in[2, n-1]$

$$
\left\{\begin{aligned}
h_{1, n} & =-2 M_{1, n} \\
h_{i, j} & =-2 M_{i, j} \text { for } \quad i \neq j \in[2, n] \\
h_{i, i} & =-M_{i, i} \\
k_{1, j} & =g_{1}-\gamma_{1} \\
k_{l, j} & =-N_{l, j}
\end{aligned}\right.
$$

Remark 28 In the same way as for Remark 22; in the $(n-1)$ first dynamics of normal form (11) we have at most $\frac{n(n-1)}{2}+1$ normal quadratic numbers $x_{i} x_{j}$ and $(n-1)^{2}$ normal quadratic numbers $x_{i} u$.

Thus, the number of normal quadratic numbers is:

$$
\begin{aligned}
\frac{n(3 n-5)}{2}+2= & (n-1) \frac{n(n+1)}{2}-(n-2) \frac{n(n+1)}{2} \\
& -(n-1)+n(n-1)-(n-1) .
\end{aligned}
$$

Now, in order to highlight the usefulness of normal form in the observability analysis and the observer design, we chose to deal with the following bio-reactor dynamics.

Example 29 Consider the following bio-reactor $d y$ namics [R]:

$$
\left\{\begin{array}{l}
\dot{\xi}=\left(\begin{array}{c}
1 \\
\frac{-1}{k}
\end{array}\right) \mu\left(\xi_{2}\right) \xi_{1} \\
y=\xi_{1}
\end{array}\right.
$$


where state $\xi=\left(\xi_{1}, \xi_{2}\right)^{T}$ represent respectively the concentration of biomass $\left(\xi_{1}\right)$ and of substrate $\left(\xi_{2}\right)$, and the $\mu\left(\xi_{2}\right)=r \xi_{2}\left(1-\frac{\xi_{2}}{c}\right)$ is the growing function. In this example, the interesting equilibrium point with respect to the observability singularity is not at $\xi=$ $(0,0)$ but at $\xi_{0}=(0, c)^{T}$.

After changing coordinate $z=\xi-\xi_{0}$, we obtain:

$$
\left\{\begin{array}{l}
\dot{z}_{1}=-r z_{1} z_{2}+O^{3}(z) \\
\dot{z}_{2}=\frac{r}{k} z_{1} z_{2}+O^{3}(z) \\
y=z_{1}
\end{array}\right.
$$

This system is not linearly observable. Using the above notations

$$
\begin{aligned}
A & =\left(\begin{array}{cc}
0 & 0 \\
0 & 0
\end{array}\right), \theta_{1}=\left(\begin{array}{cc}
\beta_{1,1} & \frac{r}{2} \\
\frac{r}{2} & 0
\end{array}\right) \\
\text { and } \theta_{2} & =\left(\begin{array}{cc}
\beta_{1,2} & -\frac{r}{2 k} \\
-\frac{r}{2 k} & 0
\end{array}\right),
\end{aligned}
$$

then the characteristic matrix of the first dynamic is

$$
M=\left(\begin{array}{cc}
\beta_{1,1} & \frac{r}{2} \\
\frac{r}{2} & 0
\end{array}\right) \text { with } \beta_{1,1}=0
$$

and normal quadratic numbers are:

$$
h_{1,2}=-r \quad \text { and } \quad h_{2,2}=0 .
$$

For the last dynamic we obtain:

$$
l_{1,2}=\frac{r}{k} \quad \text { and } \quad l_{2,2}=0 .
$$

Then, observable normal form associated to the bioreactor is:

$$
\left\{\begin{array}{l}
\dot{x}_{1}=-r x_{1} x_{2}+O^{3}(x) \\
\dot{x}_{2}=\frac{r}{k} x_{1} x_{2}+O^{3}(x) \\
y=x_{1}
\end{array}\right.
$$

In $[R]$ a solution was proposed, here our purpose is just to highlight the efficiency of the observable normal form, for this we propose the following sliding mode observer:

$$
\left\{\begin{array}{l}
\dot{\hat{x}}_{1}=-r x_{1} \hat{x}_{2}+\lambda_{1} \operatorname{sign}(y-\hat{y})+\beta(y-\hat{y})^{3} \\
\hat{\hat{x}}_{2}=\frac{r}{k} \hat{x}_{2} x_{1}+E_{1} \lambda_{2}\left(\tilde{x}_{2}-\hat{x}_{2}\right)
\end{array}\right.
$$

where $\tilde{x}_{2}=\hat{x}_{2}+E_{1} E_{s} \frac{\lambda_{1} \operatorname{sign}(y-\hat{y})}{r x_{1}}, \hat{y}=\hat{x}_{1}$ and if $\left|x_{1}\right|<\varepsilon$ then $E_{1}=0$ else $E_{1}=1$, in the same way if $e_{1}=x_{1}-\hat{x}_{1}$ is not on the constrained manifold $\left(e_{1}=0\right)$ then $E_{2}=0$ else $E_{2}=1$. Moreover, $\beta$, $\lambda_{1}$ and $\lambda_{2}$ are chosen in order to ensure at least the stability. However using the Remark 15, the submanifold observability singularity is $S_{1}=\{x \in U$, such that $\left.h_{1,2} x_{1}=0\right\}$. So, the asymptotic stability is not guaranteed for $x_{1}(t)=0$ for all $t$, then in order to take into account, we set $E_{s}=1$ if $x_{1} \neq 0$ else $E_{s}=0$.

This example shows that the observability singularity can be overcome thanks to quadratic normal form and its normal quadratic numbers which may contain information for the observer design. Here, observation information is given by $r x_{1} x_{2}$ (the term $h_{1, n} x_{1} x_{n}$ of the normal form).

\section{Conclusion}

In this paper, for nonlinear systems linearly observable and nonlinear systems with one linear unobservable mode, a particular quadratic normal form is given for observer design and detectability analysis around an equilibrium point. Using Poincaré's normal forms, we point out normal quadratic numbers that ensure the local observability. Moreover, in order to analyze the observability without transforming the systems in their normal forms, we have introduced what we have called characteristic matrices. Some of them are linked to the quadratic part of the drift and they characterize the quadratic observability without input. Others are linked to the so-called universal input and define the choice of this input to preserve the observability. These matrices inform us about the quadratic observability before we begin the design of an observer. There are many areas where this study may be implemented; the synchronization of chaotic systems $[\mathrm{NM}]$, sensorless control of induction motors [CYBMM].

Nevertheless, some of these cases need an extra development of our method. For the synchronization of chaotic systems [BBBT2, BBBT3] some modifications to the output-injection will be considered and for the sensorless control of induction motors a generalization of the approach to multi-output systems will be necessary.

\section{References}

[BBBT1] L. Boutat-Baddas, D. Boutat, J-P. Barbot and R. Tauleigne, "Quadratic Observability normal form", In Proc. of IEEE CDC 01, 2001.

[BBBT2] L. Boutat-Baddas, J.P. Barbot, D. Boutat, R. Tauleigne "Observability bifurcation versus observing bifurcations ", Proc. of the 15 th IFAC, 2002.

[BBBT3] L. Boutat-Baddas, J.P. Barbot, D. Boutat and R. Tauleigne, "Sliding mode observers 
and observability singularity in chaotic synchronization", Mathematical Problems in Engineering, Theory, Methods, and Aplication, Number 1, Volume 2004.

[BB] D. Boutat and J.P. Barbot, "Poincaré normal form for a class of driftless systems in a one dimensional submanifold neighborhood", Mathematics of Control, Signals and Systems, Vol. 15, pp. 256-274, 2002.

[B] P. Brunovsky, "A classification of linear controllable systems", Kybernetika, Vol. 6, pp. 173-188 1970.

[CYBMM] C. Canudas De Wit, A. Youssef, J.P. Barbot, Ph. Martain and F.Malrait "Observability conditions of Induction motors at Low Frequencies", Conference on Decision and Control Sydney, Australia, Dec, 2000.

[DD] H. Dang-Vu and C. Delcarte, "Bifurcation et chaos", Ellipse Edition Marketing S.A., 2000 .

[GB] J.P. Gauthier and G. Bornard, "Observability for any $u(t)$ of a class of bilinear systems", IEEE TAC, Vol. 26, pp. 922-926, 1981.

[G] P. Glendinnig "Stability, Instability and Chaos", Cambridge University Press, 1994.

[HBMN] B. Hamzi, J-P. Barbot, S. Monaco and D. Normand-Cyrot, "Nonlinear discrete-time control of systems with a Naimar-Sacker bifurcation", Systems and Control Letters 44, pp. 245-258, 2001.

[K] W. Kang, "Bifurcation and normal form of nonlinear control system: Part I and II", SIAM J. Control and Optimization, Vol. 36, pp .193-232, 1998.

[KK1] W. Kang and A.J. Krener, "Extended quadratic controller normal form and dynamic state feedback linearization of non linear systems", SIAM J. Control and Optimization, Vol. 30, No. 6, pp. 1319-1337, 1992.

[KK2] N. Kazantzis and C. Kravaris, "Nonlinear observer design using Lyapunov's auxiliary Theorem", Systems and Control Letters, Vol. 34, pp. 241-247, 1998.
$[\mathrm{K}] \quad$ A. Krener, "Approximate linearization by state feedback and coordinate change", Systems and Control Letters, Vol. 5, pp. 181-185, 1984.

[KK3] A. Krener and W.Kang, "Degree two normal forms of control systems and the generalized Legendre-Clebsh Condition", $p p$. 295-303. Analysis of Controlled Dynamical Systems, B. Bridge, J.P. Gauthier and I. Kupka, eds, Birkhauser, 1991.

[KR] A. J. Krener and W. Respondek, "Nonlinear observer with linearisable error dynamique", SIAM Journal of Control and Optimisation, Vol. 23,pp. 197-216, 1985.

[KI] A. Krener and A. Isidori, "Linearization by output injection and nonlinear observer", Systems and Control Letters, Vol. 3, pp. 47-52, 1983.

$[\mathrm{KX}] \quad$ A. Krener and M. Q Xiao, "Nonlinear observer design in the Siegel domain through coordinate changes", In Proc of the 5th IFAC Symposium, NOLCOS01, Saint-Petersburg, Russia, pp. 55\%562, 2001.

[L] A.M. Lyapunov, "Stability of Motion", Academic Press, New York and London, 1966.

$[\mathrm{NM}] \quad$ H. Nijmeijer and I.M.Y. Mareels, "An observer looks at synchronization", IEEE Trans. on Circuits and Systems-1: Fundamental Theory and Applications, Vol. 44, No 11, pp. 882-891, 1997.

[P] H. Poincaré, "Solutions périodiques dans le voisinage d'un point d'équilibre-lunes sans quadrature", dans Les Méthodes Nouvelles de la Mécanique Céleste, Gauthier-Villars: Paris, Vol. 1, pp. 156-161, 1892.

[R] A. Rapaport and A. Maloum, "Embedding For Exponential Observers Of Nonlinear Systems", In 39th CDC Conference. CDROM, 2000.

[W] S. Wiggins, "Introduction to Applied Nonlinear Dynamical Systems and Chaos", Springer, Texts in Applied Mathematics 2, 1990. 